\title{
BMJ Open Asthma and lung cancer, after accounting for co-occurring respiratory diseases and allergic conditions: a systematic review protocol
}

\author{
Rachel Denholm, ${ }^{1}$ Elizabeth Crellin, ${ }^{1}$ Ashwini Arvind, ${ }^{2}$ Jennifer Quint ${ }^{2}$
}

To cite: Denholm $R$, Crellin E, Arvind A, et al. Asthma and lung cancer, after accounting for cooccurring respiratory diseases and allergic conditions: a systematic review protocol. BMJ Open 2017;7:e013637.

doi:10.1136/bmjopen-2016013637

- Prepublication history for this paper is available online. To view these files please visit the journal online (http://dx.doi.org/10.1136/ bmjopen-2016-013637).

Received 27 July 2016 Revised 11 November 2016 Accepted 1 December 2016

CrossMark

\footnotetext{
${ }^{1}$ Department of Non-Communicable Epidemiology, London School of Hygiene and Tropical Medicine, London, UK

${ }^{2}$ Faculty of Medicine, National Heart \& Lung Institute, Imperial College London, London, UK
}

Correspondence to Dr Rachel Denholm; rachel.denholm@Ishtm.ac.uk

\section{ABSTRACT}

Introduction: Asthma is one of the most frequently diagnosed respiratory diseases in the UK, and commonly co-occurs with other respiratory and allergic diseases, such as chronic obstructive pulmonary disease (COPD) and atopic dermatitis. Previous studies have shown an increased risk of lung cancer related to asthma, but the evidence is mixed when accounting for co-occurring respiratory diseases and allergic conditions. A systematic review of published data that investigate the relationship between asthma and lung cancer, accounting for co-occurring respiratory and allergic diseases, will be conducted to investigate the independent association of asthma with lung cancer.

Methods and analysis: A systematic review will be conducted, and include original reports of cohort, cross-sectional and case-control studies of the association of asthma with lung cancer after accounting for co-occurring respiratory diseases. Articles published up to June 2016 will be included, and their selection will follow the Preferred Reporting Items for Systematic Reviews and Meta-Analyses (PRISMA) guidelines. A standardised data extraction form will be developed and pretested, and descriptive analyses will be used to summarise the available literature. If appropriate, pooled effect estimates of the association between asthma and lung cancer, given adjustment for a specific co-occurring condition will be estimated using random effects models. Potential sources of heterogeneity and between study heterogeneity will also be investigated.

Ethics and dissemination: The study will be a review of published data and does not require ethical approval. Results will be disseminated through a peerreviewed publication.

Trial registration number: International Prospective Register for Systematic Reviews (PROSPERO) number CRD42016043341

\section{INTRODUCTION}

Asthma has been shown to be associated with lung cancer risk. ${ }^{1}$ However, asthma commonly co-occurs with other respiratory

\section{Strengths and limitations of this study}

- Broad search strategy to identify a range of studies that have investigated the association between asthma co-occurring respiratory diseases and allergic conditions and lung cancer.

- The review will focus on a wide-range of co-occurring diseases that explain some potential aetiological pathways linking asthma and lung cancer.

- Use of Preferred Reporting Items for Systematic Reviews and Meta-Analyses-Protocol guidelines, tested tools for data extraction and assessment of data quality, and risk of bias analysis.

- This review is not unique in the sense that other researchers have examined the evidence linking asthma and lung disease. However, this review will further previous work by only including studies that have accounted for co-occurring respiratory and allergic conditions, and investigating heterogeneity by study and patientspecific factors.

diseases, such as chronic obstructive pulmonary disease $(\mathrm{COPD})^{2}{ }^{3}$ which is strongly associated with an increased risk of lung cancer, ${ }^{2-4}$ and allergic conditions, such as rhinitis and atopic dermatitis ${ }^{5}{ }^{6}$ whose relationship with lung cancer is less clear. ${ }^{7} 8$ The association between asthma and lung cancer after accounting for co-occurring respiratory and allergic diseases is unclear.

Asthma is one of the most frequently diagnosed respiratory diseases in the UK. A recent study of national trends found that lifetime prevalence of asthma ranges from $21.5 \%$ in $>65$ year age group to $27.7 \%$ in 45-64 year olds. ${ }^{9}$ Asthma commonly co-occurs with other respiratory diseases, in particular COPD. ${ }^{10} 11$ The overlap between asthma and COPD diagnoses can reach $20 \%$ of all patients with chronic respiratory disease. ${ }^{12}$ Diagnoses of multiple respiratory diseases increases with age, with up to $50 \%$ 
of patients with obstructive lung disease aged $\geq 50$ years being diagnosed with combined asthma and COPD. $^{13}$

As well as other respiratory diseases, it has been wellestablished that asthma, and in particular atopic asthma, co-occurs with other allergic conditions, such as allergic rhinitis (hay fever) ${ }^{14}$ and atopic dermatitis (eczema). ${ }^{15}$ In children with asthma, co-occurrence with rhinitis and/or atopic dermatitis has been reported to be as high as $\sim 44 \%{ }^{16}$ There is mixed evidence of the nature of the relationship between asthma and allergic conditions, with evidence of both a causal relationship ${ }^{17} 18$ and common causes ${ }^{19}$ including subsets of genes. ${ }^{20}$

Most evidence is indicative of an increased risk of lung cancer associated with a diagnoses of asthma. ${ }^{21-25}$ However, many previous studies have not accounted for co-occurring respiratory diseases, ${ }^{23-25}$ and of those that have, many have observed an attenuation in the relationship between asthma and lung cancer. ${ }^{126}{ }^{27}$ A previous meta-analysis published in 2012 conducted a sensitivity analysis restricting to studies that had adjusted for co-occurring chronic bronchitis, emphysema or COPD. Rosenberger $e t a l^{1}$ found an inverse association between asthma and lung cancer in studies that adjusted for co-occurring COPD (shown in online supplementary table V). In addition, studies that have accounted for co-occurring allergic conditions have found positive associations between asthma and lung cancer attenuate, and are no longer significant, ${ }^{28}$ or reverse and show a protective effect. ${ }^{22}$

Further investigation of the literature is needed to explore the nature of asthma's independent association with lung cancer. By further exploring this association it may be possible to disentangle the relationship between COPD, allergic conditions and asthma, and lung cancer. It is thought that by accounting for co-occurring respiratory diseases and allergic conditions, it will be possible to investigate the independent association of asthma with lung cancer, and direct further research to establish potential aetiological mechanisms.

\section{METHODS AND ANALYSIS}

Aim: The main objective of the study is to systematically review published data on the association between asthma and lung cancer risk which have accounted for the presence of other respiratory diseases and allergic conditions.

Specific aims are:

1. To examine associations between asthma and lung cancer after adjustment for co-occurring respiratory and allergic diseases, specifically

I. COPD (including chronic bronchitis and emphysema)

II. Bronchiectasis

III. Pulmonary fibrosis

IV. Tuberculosis
V. Pneumonia
VI. Allergic rhinitis
VII. Atopic dermatitis (eczema)
VIII. Urticarial
IX. Anaphylaxis
$\mathrm{X}$. Conjunctivitis
XI. Food allergy

2. To identify sources of heterogeneity in study-specific estimates.

Inclusion criteria ( participants, interventions, comparisons and outcomes):

Participants: Adults $\geq 18$ years old.

Eligible studies: We will include original reports (primary data collection) including cohort, crosssectional and case-control studies that have investigated the relationship between asthma and lung cancer and included at least one other respiratory or allergic disease in the model, or restricted the exposure to patients with asthma 'only' and no other co-occurring respiratory or allergic condition.

Outcome: The primary outcome of interest will be confirmed lung cancer case (either through cancer registry or administration records), International Classification of Diseases 10th Revision code C34. If included, subtypes of lung cancer will also be included.

\section{Exclusion criteria}

We will exclude the following literature identified in the database searchers: conference abstracts and proceedings, case studies, reviews and general discussion papers; studies that investigate asthma and lung cancer but do not account for any co-occurring conditions in the study design or analysis. We will also exclude unpublished literature and ongoing studies from the review.

\section{Literature search}

We will search PubMed, EMBASE, MEDLINE, ISI Web of Science and the Cumulative Index to Nursing and Allied Health up to 30 June 2016 using medical subject headings and text words related to asthma, lung cancer and respiratory diseases. Reference lists of all included studies will be cross-checked to identify other potentially relevant studies. In addition, reference lists of reviews and discussion articles-which will be ineligible for the review-will be searched and cross-checked for potential relevant manuscripts that have been missed by the database searches.

The results and dates of each search will be recorded. Search terms:

("lung cancer" OR "malignant neoplasm lung" OR "pulmonary cancer") OR "lung neoplasm" (MeSH Term)

AND

"Asthma*" (MeSH Term) OR ("asthm*" OR "Asthma”) 
AND

OR

("Pulmonary disease, chronic obstructive" (MeSH Term) OR "Chronic obstructive airway disease" [MeSH Term) OR "Chronic obstructive lung disease " (MeSH Terms) OR "Chronic obstructive pulmonary disease" (MeSH Term))

\section{OR}

("Bronchitis*" (MeSH Term) OR "Emphysema*" (MeSH Term) OR "Bronchiectasis*" (MeSH Term) OR "Idiopathic pulmonary fibrosis" (MeSH Term) OR "Pulmonary fibrosis*" (MeSH Term) OR "Latent tuberculosis infection" (MeSH Term) OR tuberculosis* (MeSH Term) OR pneumonia* (MeSH Term))

OR

("chronic obstructive pulmonary disease" OR "COPD" OR "bronchitis" OR "chronic bronchitis" OR "emphysema" OR "pulmonary disease" OR "pulmonary emphysema" OR "lung disease, obstructive" OR"bronchiectasis" OR "pulmonary fibrosis" OR "Tuberculosis" OR Pneumonia")

OR

(Rhinitis, Allergic*" (MeSH Term) OR "Hay fever" (MeSH Term)) OR ("hay fever" OR "allergic rhinitis")

OR

("(Dermatitis, Allergic Contact” (MeSH Term) OR "Allergic Contact Dermatitis" (MeSH Term) OR "Dermatitis, Allergic Eczematous" (MeSH Term) OR "Dermatitis, Contact, Allergic" (MeSH Term) OR "Atopic eczema" (MeSH) OR "Eczema*" (MeSH Term)) OR ("atopic dermatitis" OR "atopic eczema" OR “eczema*)

OR

"Urticaria" (MeSH Term) OR ("urticaria*” OR "hives" OR "welts" OR "weals")

OR

("Anaphylaxis" (MeSH Term) OR "Shock" (MeSH Term)) OR "anaphylaxis"

OR

"Conjunctivitis, Allergic" (MeSH Term) OR ("conjunctivitis" OR "red eye")

\section{OR}

("Food hypersensitivity" (MeSH Term)) OR ("food sensitivity" OR "food allergy" OR "food intolerance" OR "angioedema")

\section{Selection of studies and extraction of data}

Literature searches of the electronic databases listed above will be conducted and the resulting citations will be downloaded to EndNote software, where duplicate citations will be removed. Any additional citations identified through manual searches will be added to this database.

The titles and abstracts from this initial database will be screened by two reviewers (AA and RD) and classified using the eligibility and exclusion criteria described above as:

- Yes, full paper to be retrieved and screened

- No, exclude

- Unclear

Two reviewers will independently review the full-text references classified as 'Yes' or 'Unclear' and assess eligibility to be included in the review. Difference in opinion will be resolved by consensus, or through arbitration by a third reviewer.

\section{Data abstraction}

A standardised data extraction form will be developed and pretested. Any ambiguities will be discussed and the form amended accordingly.

Data will be extracted on the following variables:

- Study identifiers: ID, author(s), year of publication;

- Characteristics of the study population: country, study design (eg, cohort, case-control population-based), study period, eligibility criteria, recruitment and participation rates, and final sample size;

- Participant characteristics: sex, age-group and ethnicity;

- Co-occurring disease: named other respiratory or allergic diseases included in the model;

- Source of exposure/outcome data: self-reports, selfreport of doctors diagnosis, doctor/hospital record;

- Adjustment for other confounding factors: smoking status, age, medication, sex and deprivation;

- Final adjusted results for each particular study.

If there are multiple eligible papers from the same study only the one based on the largest sample size, or the one with the most comprehensive exposure data, will be selected for inclusion in the systematic review. For published papers based on pooled studies, data will be extracted for each individual study and included as an independent study in the meta-analysis. The process of literature selection and reasons for exclusion will be fully documented and a PRISMA ${ }^{29}$ flow-diagram will be constructed.

\section{Study quality assessment}

Additional data extracted from each study will be extracted to assess quality using a specifically developed standardised quality assessment form. This assessment form will be developed to capture three domains:

- Potential for selection bias (eg, study design; participation rates; percentage of the study population with both exposure and outcome data); 
- Potential for exposure and outcome measurement errors (eg, source of exposure/outcome data);

- Availability of data on key variables (eg, smoking status).

A list of items for each one of the three domains will be developed. For each item, papers will be allocated a score ranging from 0 (if it does not meet the criteria or if the information provided is unclear) to a maximum to be defined (eg, 1, 4 or 8 , depending on the specific item). The overall quality of the study will be expressed as the sum of its item-specific scores. The higher the score the higher the methodological quality of the study, that is the lower the risk that its findings may have been affected by bias. In addition, each study will receive a global quality rating using the Effective Public Health Practice Project's Quality Assessment Tool for Quantitative Studies and analysis will be conducting using both study quality methods.

\section{Data analysis}

The extracted data will be analysed in STATA (Statistical Software V.14 (StataCorp, Texas, USA).

Basic descriptive analyses will be conducted to summarise information about the study population (eg, by country, type of study), source of exposure/outcome, co-occurring diseases etc.

Analyses will be conducted separately for each exposure. If appropriate, depending on the number and characteristics of the studies included in the review, and on the data reported, pooled effect estimates of the association between asthma and lung cancer, given adjustment for a specific co-occurring respiratory or allergic disease, for example, COPD will be estimated using random effects models. For each exposure, sensitivity analysis will take into account whether other additional respiratory or allergic disease have been accounted for in the analysis.

To examine potential sources of heterogeneity, studyspecific estimates will be stratified according to relevant factors (eg, smoking status) and methodologically relevant variables (eg, source of exposure data; study design; study quality score).

Between-study heterogeneity will be formally assessed using I2. ${ }^{30}$ The findings will be tabulated and/or displayed graphically using forest plots.

Small study bias will be assessed via funnel plots and the Egger funnel plot asymmetry test. ${ }^{31}$

Contributors RD and JQ conceived the study. RD drafted the original protocol, which was reviewed by all the authors. All authors approved the final protocol for publication. RD acts as guarantor of the work. EC and AA will undertake screening, data extraction and data synthesis.

Competing interests None declared.

Provenance and peer review Not commissioned; externally peer reviewed.

Open Access This is an Open Access article distributed in accordance with the Creative Commons Attribution Non Commercial (CC BY-NC 4.0) license, which permits others to distribute, remix, adapt, build upon this work noncommercially, and license their derivative works on different terms, provided the original work is properly cited and the use is non-commercial. See: http:// creativecommons.org/licenses/by-nc/4.0/

\section{REFERENCES}

1. Rosenberger A, Bickeböller H, McCormack V, et al. Asthma and lung cancer risk: a systematic investigation by the International Lung Cancer Consortium. Carcinogenesis 2012;33:587-97.

2. Brenner DR, Boffetta P, Duell EJ, et al. Previous lung diseases and lung cancer risk: a pooled analysis from the International Lung Cancer Consortium. Am J Epidemiol 2012;176:573-85.

3. Wu AH, Fontham ETH, Reynolds $\mathrm{P}$, et al. Previous lung disease and risk of lung cancer among lifetime nonsmoking women in the United States. Am J Epidemiol 1995;141:1023-32.

4. Liang $\mathrm{H}$, Guan $\mathrm{P}$, Yin Z, et al. Risk of lung cancer following nonmalignant respiratory conditions among nonsmoking women living in Shenyang, Northeast China. J Womans Health (Larchmt) 2009;18:1989-95.

5. Arabkhazaeli A, Vijverberg SJ, van Erp FC, et al. Characteristics and severity of asthma in children with and without atopic conditions: a cross-sectional study. BMC Pediatr 2015;15:172.

6. Bieber T. Atopic dermatitis. N Engl J Med 2008;358:1483-94.

7. Hemminki K, Försti $A$, Fallah $M$, et al. Risk of cancer in patients with medically diagnosed hay fever or allergic rhinitis. Int $J$ Cancer 2014;135:2397-403.

8. Wang $\mathrm{H}$, Rothenbacher $\mathrm{D}$, Low $\mathrm{M}$, et al. Atopic diseases, immunoglobulin $E$ and risk of cancer of the prostate, breast, lung and colorectum. Int J Cancer 2006;119:695-701.

9. Simpson CR, Sheikh A. Trends in the epidemiology of asthma in England: a national study of 333,294 patients. J $R$ Soc Med 2010;103:98-106.

10. de Marco R, Pesce G, Marcon A, et al. The coexistence of asthma and chronic obstructive pulmonary disease (COPD): prevalence and risk factors in young, middle-aged and elderly people from the general population. PLOS ONE 2013;8:e62985.

11. Soriano JB, Visick GT, Muellerova $\mathrm{H}$, et al. Patterns of comorbidities in newly diagnosed COPD and asthma in primary care. Chest 2005;128:2099-107.

12. Miravitlles M, Andreu I, Romero $\mathrm{Y}$, et al. Difficulties in differential diagnosis of COPD and asthma in primary care. Br J Gen Pract 2012;62:e68-75.

13. Soriano JB, Davis KJ, Coleman B, et al. The proportional Venn diagram of obstructive lung disease: two approximations from the United States and the United Kingdom. Chest 2003;124:474-81.

14. Guerra S, Sherrill DL, Martinez FD, et al. Rhinitis as an independent risk factor for adult-onset asthma. J Allergy Clin Immunol 2002;109:419-25.

15. Ronmark EP, Ekerljung L, Lotvall J, et al. Eczema among adults: prevalence, risk factors and relation to airway diseases. Results from a large-scale population survey in Sweden. Br J Dermatol 2012;166:1301-8

16. Garcia-Aymerich J, Benet M, Saeys $Y$, et al. Phenotyping asthma, rhinitis and eczema in MeDALL population-based birth cohorts: an allergic comorbidity cluster. Allergy 2015;70:973-84.

17. Shaaban R, Zureik M, Soussan D, et al. Rhinitis and onset of asthma: a longitudinal population-based study. Lancet 2008;372:1049-57.

18. Martin PE, Matheson MC, Gurrin L, et al. Childhood eczema and rhinitis predict atopic but not nonatopic adult asthma: a prospective cohort study over 4 decades. J Allergy Clin Immunol 2011;127:1473-9 e1.

19. Rimmer J, Ruhno JW. 6: Rhinitis and asthma: united airway disease Med J Aust 2006;185:565-71.

20. Heinzmann A, Deichmann KA. Genes for atopy and asthma. Curr Opin Allergy Clin Immunol 2001;1:387-92.

21. Boffetta $P, Y e$ W, Boman $G$, et al. Lung cancer risk in a population-based cohort of patients hospitalized for asthma in Sweden. Eur Resp J 2002;19:127-33.

22. Hwang $\mathrm{CY}$, Chen YJ, Lin MW, et al. Cancer risk in patients with allergic rhinitis, asthma and atopic dermatitis: a nationwide cohort study in Taiwan. Int J Cancer 2012;130:1160-7.

23. Ji J, Shu X, Li X, et al. Cancer risk in hospitalised asthma patients. Br J Cancer 2009;100:829-33.

24. Turner MC, Chen Y, Krewski D, et al. Cancer mortality among US men and women with asthma and hay fever. Am J Epidemiol 2005;162:212-21

25. Vesterinen E, Pukkala E, Timonen T, et al. Cancer incidence among 78,000 asthmatic patients. Int J Epi 1993;22:7. 
26. Denholm R, Schüz J, Straif $\mathrm{K}$, et al. Is previous respiratory disease a risk factor for lung cancer? Am J Resp Crit Care Med 2014;190:549-59.

27. Powell HA, Iyen-Omofoman B, Baldwin DR, et al. Chronic obstructive pulmonary disease and risk of lung cancer: the importance of smoking and timing of diagnosis. J Thorac Oncol 2013;8:e34-5.

28. Gorlova OY, Zhang Y, Schabath MB, et al. Never smokers and lung cancer risk: a case-control study of epidemiological factors. Int J Cancer 2006;118:1798-804.
29. Swartz MK. The PRISMA statement: a guideline for systematic reviews and meta-analyses. J Pediatr Health Care 2011;25:1-2.

30. Higgins JP, Thompson SG, Deeks JJ, et al. Measuring inconsistency in meta-analyses. BMJ 2003;327: 557-60.

31. Egger M, Davey Smith G, Schneider M, et al. Bias in meta-analysis detected by a simple, graphical test. BMJ 1997;315:629-34. 